\title{
A fuzzy development for attribute control chart with Monte Carlo simulation method
}

\author{
Mohammad Hadi Madadi ${ }^{\mathrm{a}}$ and Morteza Mahmoudzadeh ${ }^{\mathrm{b} *}$
}

andustrial Engineering Department, Non-Governmental and Private Higher Education Institution, Alghadir, Tabriz, Iran ${ }^{b}$ Department of Management, Science and Research Branch, Islamic Azad University, Tabriz, Iran

\section{H R O N I C L E}

\section{Article history:}

Received: February 1, 2017

Received in revised format: July

6,2017

Accepted: July 31, 2017

Available online:

August 2, 2017

Keywords:

Statistical process control

Fuzzy logic

Membership function

Simulation

Multinomial distribution

\section{A B S T R A C T}

This paper presents the case study of fuzzy statistical process control which has been simulated for variable and discontinuous production within a particular time frame in a key manufacturing workshop. In order to reduce waste production and increase productivity, dimensional inspection from raw product is categorized into three groups: product of type A, product of type B, and discard. In first part, the appearance characteristics of product is defined as fuzzy membership function as the input of the system in order to allocate the output obtained from fuzzy inference of product to one of the three quality levels. Afterwards, each quality level is assigned to its own group by means of Monte Carlo simulation techniques. In the second part, with fuzzy development of a multinomial $p$ chart, the production process is illustrated as a control chart within the particular period of time.

\section{Introduction}

Quality has always been considered as an essential part of products or services. A product or service failures is normally detected through quality control (Webber \& Wallace, 2011). The statistical methods such as Statistical Process Control (SPC) play an essential role to play for the quality improvement (Cheng, 2005). SPC is a strong set of problem-solving tools to facilitate achieving process stability and improving capability via the reduction of variability (Montgomery, 2009).

Control charts, one of quality control tools, are the most significant tool implemented by SPC for process control (Misra, 2008). According to Montgomery (2009), these charts are mostly applied for online surveillance which results in process improvement.

Generally, control charts can be divided into two categories; control charts for variables and control charts for attributes (Lighter \& Fair, 2000). Variables are measurable quality characteristics, such as dimension, weight, or volume and also they can be presented in terms of numerical measurement units (Montgomery, 2009). However, some of the quality characteristics that cannot be measured numerically are named attributes. Attributes are measured as either "acceptable" or "not acceptable" (Fryman,

* Corresponding author

E-mail address: m.mahmoudzadeh206@gmail.com (M. Mahmoudzadeh) 
2002). In order to reduce waste production and increase profitability, those parts that are not accepted in quality test can be recycled or turned to another similar products. In this stage, a number of quality levels are defined between two categories of "acceptable" and "not acceptable" (Ross, 2009). For example, the quality of a product can be classified into three grades; grade 1, grade 2, and grade 3 (Raz $\&$ Wang, 1990). Increasing the number of quality characteristics can be resulted in major increase in distortion of the process-monitoring procedure (Montgomery, 2009). Except for very special cases, fuzzy control charts are used instead of attributes control charts such as $p$ or $c$ charts (Kahraman, 2006).

Fuzzy set theory has proven its great ability for representing vague data and also fuzzy logic proffers a systematic base to handle situations, which are ambiguous or not well defined (Gülbay \& Kahraman, 2007). In 1965, the term "fuzzy sets" was introduced by Zadeh as an approach for modeling human reasoning under uncertainly (Du \& Swamy, 2006). Fuzzy approach transforms the crisp values into the linguistic terms, creates input fuzzy sets, analyzes with the help of if-then rules to form output fuzzy sets, and then, produces definitive data through defuzzification process (Ross, 2009).

Many studies have been performed on the application of fuzzy approach in quality control. For example, the fuzzy set theory was applied by Bradshaw (1983) as a basis for interpreting the representation of a graded degree of product conformance. He placed emphasis on the advantages of fuzzy economic control limits over traditional acceptance charts (Bradshaw, 1983). In order to overcome the binary classification limits of attribute control charts, Wang and Raz (1990) applied linguistic terms such as "perfect", "good", "poor", etc. to have a better explanation of intermediate levels of quality compared to classical values.

Kanagawa et al. (1993) used linguistic data to explain output process and developed a new control chart for the monitoring of the mean and the deviation of attribute variables. Franceschini and Romano (1999) proposed a procedure, which depends on the use of linguistic quantifiers for constructing control charts. Triangular fuzzy numbers was successfully employed to examine the control charts for unnatural patterns by Kahraman et al. (1995). The application of fuzzy logic to vary the SPC rules was studied by El-Shal and Morris (2000). They purposed at improving the detection, detection-speed of real faults and lowering the creation of false alarms (El-Shal \& Morris, 2000). Later, based on the fuzzy set and probability theories, different tests of constructing control charts for linguistic data was explained by Taleb and Limam (2002). In order to reflect the ambiguity of data and tightness of inspection, $\alpha$-level fuzzy control chart for attributes was proposed by Gülbay and Kahraman (2004). To deal with the expert's subjective judgments, Cheng (2005) suggested a conditional technique for the ranking scores assigned by the individual inspectors to the inspected items. A p-control chart was constructed by Shu $\& \mathrm{Wu}(2010)$ to monitor the fraction of nonconforming items in the process in which fuzzy sample data are collected from the manufacturing process. A fuzzy multinomial process with Variable Sample Size (VSS) was presented by Pandurangan and Varadharajan (2011), and consequently, the control limits for the Fuzzy Multinomial chart were obtained via multinomial distribution.

In the present study, fuzzy statistical process control was applied in a practical example. Monte Carlo method was used for simulation of manufacturing process (Rubinstein \& Kroese, 2016). To this end, in addition to recording the number of daily orders, the limitations in dimensional inspection of the products quality levels was defined to produce random numbers related to the limitations. Finally, with the help of fuzzy approach and according to the general framework considered by Ross (2009), a multinomial $p$ chart was developed.

\section{Problem description}

The present study was placed in a key workshop with a diverse and variable workload. The workshop produces verity of keys for various usages. For instance, a car key is made from the raw key once an 
order is received from the customer. The prepared key can be used to open the doors, as well as start the ignition, and also open the trunk of the car.

The data have been used in the present study were collected over a period of 3 months to simulate a 25-day work process. In this study, the number of customers' orders was recorded every day and inspection process was performed on more than thousands of raw keys. The raw keys were purchased in packages of 100 or 150 pieces from the market and measured with a digital caliper. If the size of the raw keys is in a standard range, then the process of making new keys is started. In order to make a key for a type $A$ car, the desired range which has been shown by $K_{1}$ and $K_{2}$ are $(3.57 \pm 0.03 \mathrm{~mm})$ and $(5.38$ $\pm 0.03 \mathrm{~mm}$ ), respectively (Fig.1).

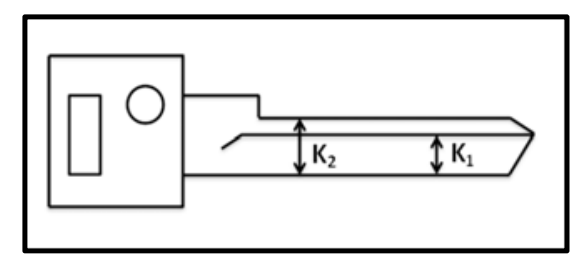

Fig. 1. Raw key

In order to decrease the culls in this workshop, the out of range keys were used for making keys for type B cars but otherwise they became culls. There are three categories for keys. Grade 1 for type A car, grade 2 for type B car and grade 3 for culls.

The statistical process control charts such as p chart, check the system status under two conditions; conforming or nonconforming (Montgomery, 2009). In the first part of the problem description, we use a fuzzy rule-base to allocate the measured keys into the appropriate categories. Furthermore, in the second part, we develop a multinomial $\mathrm{p}$ chart based on the aforementioned three categories of keys (Ross, 2009).

The fuzzy system which was used to categorize the keys consisted of two inputs and one output variables. The input variables are as follow:

1) $\boldsymbol{K}_{1}$ : a range from $3.54(\mathrm{~mm})$ to $3.63(\mathrm{~mm})$.

2) $K_{2:}$ a range from $5.35(\mathrm{~mm})$ to $5.47(\mathrm{~mm})$.

Moreover, the output variable was for grading the keys with a range from 0 to 1.2. The fuzzy rules which were obtained through interview with an expert had a form as follow:

- If (the size of $k_{1}$ ) is ... and (the size of $k_{2}$ ) is ... then (the grade of key) is ....

The results which were obtained from fuzzy inference system indicated how many orders were placed each day. These data were combined with fuzzy membership functions which describes the keys ranks after dimensional inspection. This process showed that the keys belong to which category (Ross, 2009). Afterward, each category was represented with a vector having three elements. The first, the second, and the third elements of vector were the numbers of grade 1, grade 2, and culls, respectively, relative to the total number of daily views. These vectors constituted an observation from a multivariate normal distribution (Timm, 2002). In the next step, a fuzzy representation was constructed according to these vectors (Ross, 2009), which resulted in formation of a different fuzzy distribution for each day. The $\alpha$ level fuzzy midrange was used to represent the central tendency of a fuzzy set (Cox, 2005). Finally, $p$ value, centerline, and control limits were determined for constructing a control chart. 


\section{Discussions and Results}

To create a fuzzy model, the numerical values obtained from $K_{1}$ and $K_{2}$ were defined with three linguistic variables. Moreover, the results gathered from key measurements, which showed keys with different sizes for different types of cars, were corresponded to three linguistic variables. Then each linguistic variable called fuzzy membership function was shown with a fuzzy number.

\subsection{Fuzzy Modeling}

The input and the output variables have three membership functions. Each input variable is represented by three isosceles triangles. The output variable has two right triangles on the sides and one isosceles triangle in the middle. The inputs variables of $K_{1}$ and $K_{2}$ are shown in Fig 2 and Fig 3, respectively. The membership functions of $K_{1}$ and $K_{2}$ as a $=$ Accurate, $\mathrm{b}=$ relatively accurate and $\mathrm{c}=$ Incorrect.

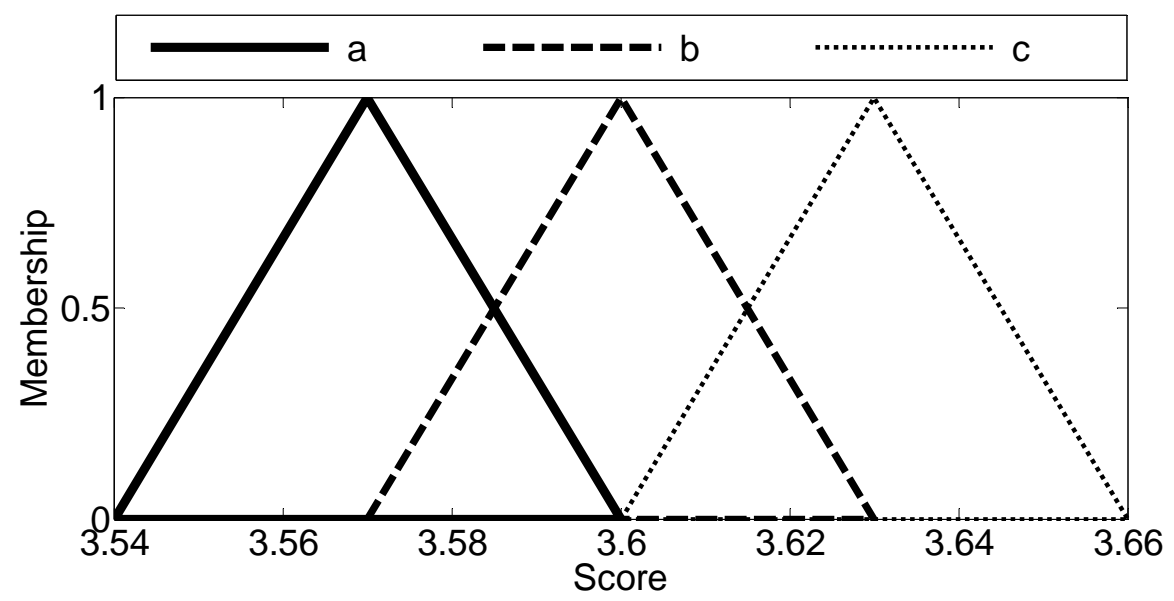

Fig. 2. Input membership functions for $K_{1}$

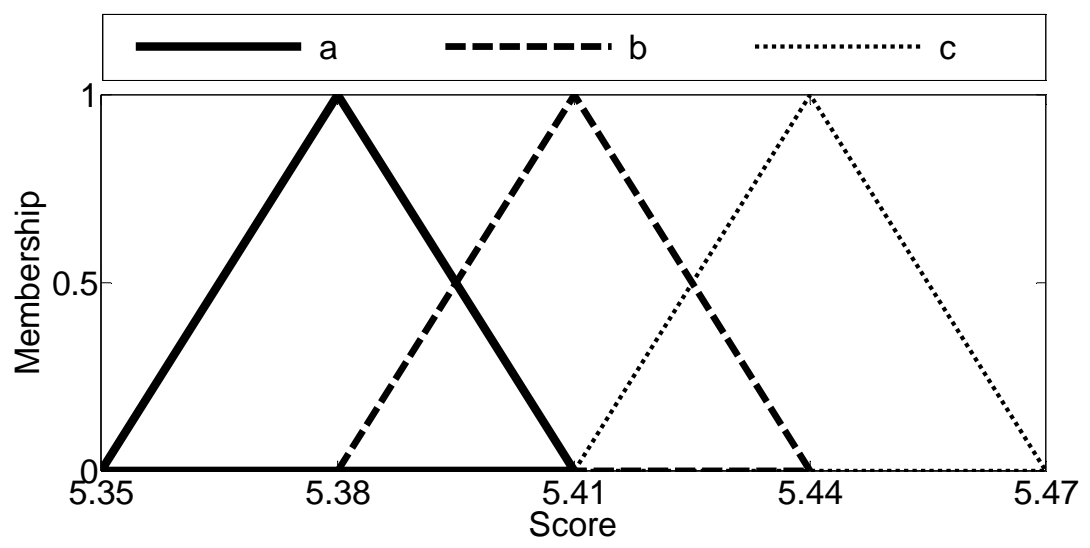

Fig. 3. Input membership functions for $K_{2}$

And the membership functions of output variable as $a=\operatorname{grade} 1, b=$ grade 2 and $c=$ grade 3 (Fig 4). 


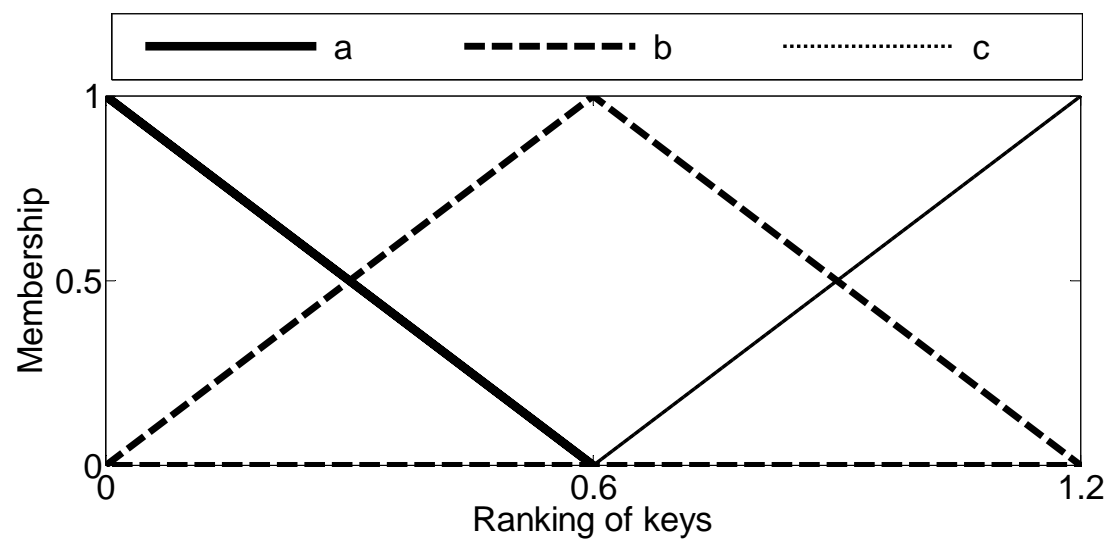

Fig. 4. Output membership functions for category keys

Each input and output variables have three membership functions. The inputs and outputs are related to each other by 9 rules $(3 \times 3)$. Fuzzy rules for this problem are presented in Table 1 .

Table 1

Fuzzy rules for category keys

\begin{tabular}{cccc}
\hline Rules & $K_{1}$ & $K_{2}$ & Grade of keys \\
\hline 1 & accurate & accurate & Grade of 1 \\
2 & accurate & relatively accurate & Grade of 1 \\
3 & accurate & Incorrect & Grade of 2 \\
4 & relatively accurate & accurate & Grade of 1 \\
5 & relatively accurate & relatively accurate & Grade of 2 \\
6 & relatively accurate & Incorrect & Grade of 2 \\
7 & Incorrect & accurate & Grade of 2 \\
8 & Incorrect & relatively accurate & Grade of 3 \\
9 & Incorrect & Incorrect & Grade of 3 \\
\hline
\end{tabular}

\subsection{Fuzzy Simulation}

According to Monte Carlo method, a domain of possible inputs which was based on the results obtained from the dimensional inspections of raw keys per package was defined. For example, 60 to 80 per cent of a package including 100 pieces of raw keys had exact dimensions in the present study. The fuzzy membership functions which describe the rank of keys after inspections are shown in Fig 5. The configuration is based on the inspection of 10 pack-ages of 100 pieces of raw keys.

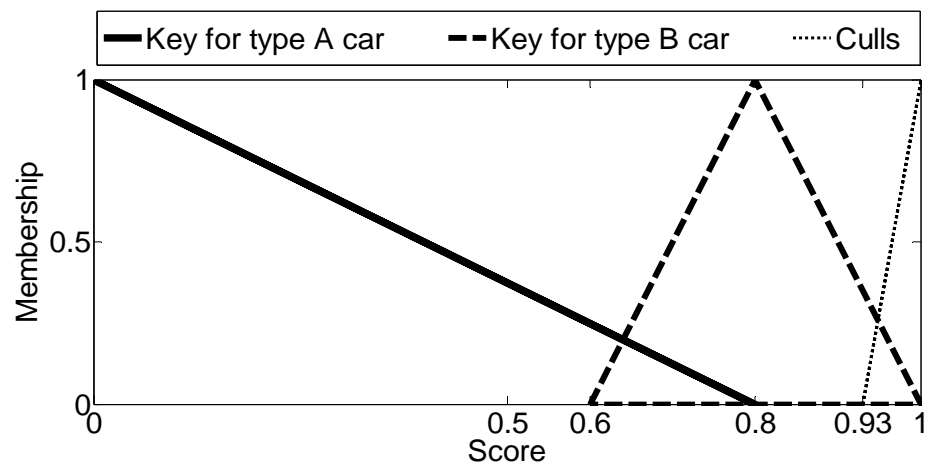

Fig. 5. Fuzzy membership function describing keys dimension after inspections 
Table 2 shows the number of orders and the measured values which were recorded for the first day. In this research, by applying the max-min Mamdani inference (Zimmermann, 2011), and the centroid method (Starczewski, 2012), the fuzzy rules were used for defuzzification of results via MATLAB software (Version: R2011b).

Table 2

Fuzzy inference system for the first day

\begin{tabular}{|c|c|c|c|c|}
\hline Day & Number of orders & $K_{1}$ & $K_{2}$ & Defuzzified values \\
\hline \multirow{7}{*}{1} & \multirow{7}{*}{7} & 3.59 & 5.40 & 0.56 \\
\hline & & 3.65 & 5.46 & 0.94 \\
\hline & & 3.62 & 5.43 & 0.73 \\
\hline & & 3.65 & 5.46 & 0.94 \\
\hline & & 3.54 & 5.35 & 0.60 \\
\hline & & 3.55 & 5.36 & 0.25 \\
\hline & & 3.57 & 5.38 & 0.19 \\
\hline
\end{tabular}

The categorization is executed by the combination of the defuzzified values based on Fig 2, and then the category with the highest membership value is picked up. For instance, the value of 0.73 in Fig 2 has the membership values of 0.08 for type A car and 0.65 for type B car. This process is repeated for the remaining days as shown in Table 3.

Table 3

Categories Keys for twenty-five days

\begin{tabular}{ccccc}
\hline Days & Number of Orders & Keys for Car type A & Keys for Car type B & Number of culls \\
\hline 1 & 7 & 4 & 3 & 0 \\
2 & 10 & 6 & 4 & 0 \\
3 & 9 & 5 & 4 & 0 \\
4 & 9 & 6 & 3 & 1 \\
5 & 5 & 3 & 1 & 0 \\
6 & 6 & 5 & 1 & 3 \\
7 & 7 & 2 & 2 & 2 \\
8 & 10 & 7 & 1 & 0 \\
9 & 6 & 4 & 2 & 2 \\
10 & 7 & 3 & 2 & 1 \\
11 & 5 & 4 & 0 & 1 \\
12 & 7 & 4 & 2 & 1 \\
13 & 7 & 6 & 0 & 1 \\
14 & 10 & 8 & 1 & 0 \\
15 & 10 & 7 & 2 & 0 \\
16 & 7 & 7 & 0 & 1 \\
17 & 10 & 6 & 4 & 0 \\
18 & 8 & 7 & 0 & 1 \\
19 & 9 & 5 & 4 & 0 \\
20 & 8 & 4 & 3 & 1 \\
21 & 7 & 5 & 2 & 0 \\
22 & 10 & 8 & 1 & 1 \\
23 & 9 & 7 & 2 & 0 \\
24 & 10 & 7 & 2 & 1 \\
25 & 8 & 7 & & 1 \\
\hline
\end{tabular}




\subsection{Fuzzy p chart}

In this section, a fuzzy multinomial control chart was formed based on the size of variable sample. The statistical principle underlying this chart was based on the multinomial distribution. The daily categories are shown with Vectors $\mathrm{X}_{1}$ to $\mathrm{X}_{25}$ :

$$
X_{1}=\left[\begin{array}{lll}
\frac{4}{7} & \frac{3}{7} & \frac{0}{7}
\end{array}\right]^{\mathrm{T}}, \ldots, X_{25}=\left[\begin{array}{lll}
\frac{7}{8} & \frac{1}{8} & \frac{0}{8}
\end{array}\right]^{\mathrm{T}}
$$

Fig 2 is defined with a matrix, called as matrix $\mathrm{Y}$, in which the columns are in the form of triangular fuzzy numbers (TFN) (Ross, 2009):

$$
Y=\left[\begin{array}{ccc}
0 & 0.6 & 0.93 \\
0 & 0.8 & 1 \\
0.8 & 1 & 1
\end{array}\right]
$$

The F1 vector is a TFN that represents the fuzzy distribution for first day. See (Fig 6):

$$
F_{1}=Y X_{1}=\left[\begin{array}{ccc}
0 & 0.6 & 0.93 \\
0 & 0.8 & 1 \\
0.8 & 1 & 1
\end{array}\right] \times\left[\begin{array}{c}
0.571 \\
0.429 \\
0
\end{array}\right]=\left[\begin{array}{c}
0.257 \\
0.343 \\
0.885
\end{array}\right]
$$

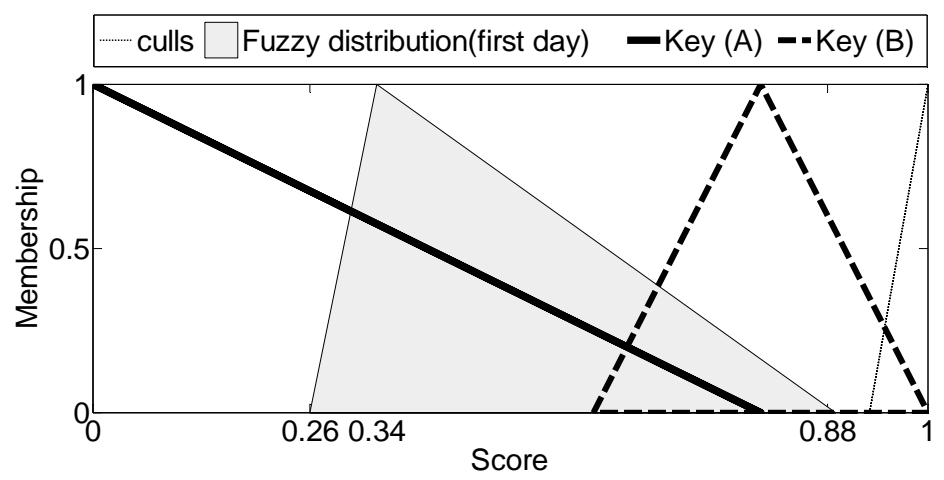

Fig. 6. The fuzzy distribution for first day

The $\alpha$-level fuzzy midrange can be represented by Eq. (1).

$$
\mathrm{P}_{\mathrm{i}}=\underline{\alpha}^{\mathrm{T}} \mathrm{YX}_{\mathrm{i}} \quad ; \quad \underline{\alpha}=\left[\begin{array}{lll}
\frac{1-\alpha}{2} & \alpha & \frac{1-\alpha}{2}
\end{array}\right]^{\mathrm{T}}
$$

For $\alpha=1 / 3$ :

$$
\mathrm{p}_{1}=\left[\begin{array}{lll}
\frac{1}{3} & \frac{1}{3} & \frac{1}{3}
\end{array}\right] \times\left[\begin{array}{l}
0.257 \\
0.343 \\
0.885
\end{array}\right]=0.495
$$

The value of 0.495 is the $P_{l}$ value or the first point on the $P$ chart. The $\pi$ vector is the mean vector of the $\mathrm{X}$ vectors (Timm, 2002) as shown in Eq. (2).

$$
\pi=\mathrm{E}(\mathrm{X})=\left[\begin{array}{c}
\mu_{1} \\
\vdots \\
\mu_{25}
\end{array}\right]
$$


$\pi=\left[\begin{array}{lll}0.681 & 0.228 & 0.09\end{array}\right]^{T}$

The mean and variance values for the first day can be represented by Eq. (3) and Eq. (4) (Ross, 2009).

$$
\begin{aligned}
& \mu=\underline{\alpha}^{\mathrm{T}} Y \pi \\
& \sigma^{2}=\underline{\alpha}^{\mathrm{T}} Y \Sigma Y^{\mathrm{T}} \underline{\alpha}
\end{aligned}
$$

For the first day: $\mu=0.452$ and $\sigma^{2}=0.0099$

According to the Ross (2009), the covariance matrix $\Sigma$ and control limits are defined by Eq. (5) and Eq. (6), because of the variable daily orders.

$$
\begin{aligned}
& \Sigma=\left[\sigma_{i j}\right]=\left\{\begin{array}{ll}
\frac{\pi_{i}\left(1-\pi_{i}\right)}{n} & , i=j \\
-\frac{\pi_{i} \pi_{j}}{n} & , i \neq j
\end{array}\right\} \\
& U_{C L} L_{p f}=\mu+Z_{c} ; \quad L L_{p f}=\mu-Z_{c}
\end{aligned}
$$

For the first day:

$$
\begin{gathered}
\Sigma=\left[\begin{array}{ccc}
0.034 & -0.034 & 0 \\
-0.034 & 0.34 & 0 \\
0 & 0 & 0
\end{array}\right] \\
\mathrm{UCL}_{\mathrm{pf}}=0.64 \\
\mathrm{LCL}_{\mathrm{pf}}=0.26
\end{gathered}
$$

In this part, $Z_{c}=1.96$ has been picked up with a confidence level of $95 \%$ (Kim, 1992). The obtained results from this process for the remaining days are given in Table 4. These data are used for drawing of a control chart which has been shown in Fig. 7. This diagram which has been drawn by EXCEL software (Version: 14.0.7182.5000). The Variable control limits are due to the different values of daily orders.

The Control chart shows that all points are within the control limits. The points are within the control limits, which indicate that $100 \%$ of the data are very close to confidence level $95 \%$. This chart provided us with an appropriate analysis of the existing condition. For instance, interestingly, the majority of inspected cases were categorized as grade 1 products in days $16^{\text {th }}$ and $25^{\text {th }}$, while as culls in day $17^{\text {th }}$. The randomness test shows that at the significance level of 0.05 with $p$-value $=0.78$, the points are randomly distributed on the graph (Bajpai, 2009).

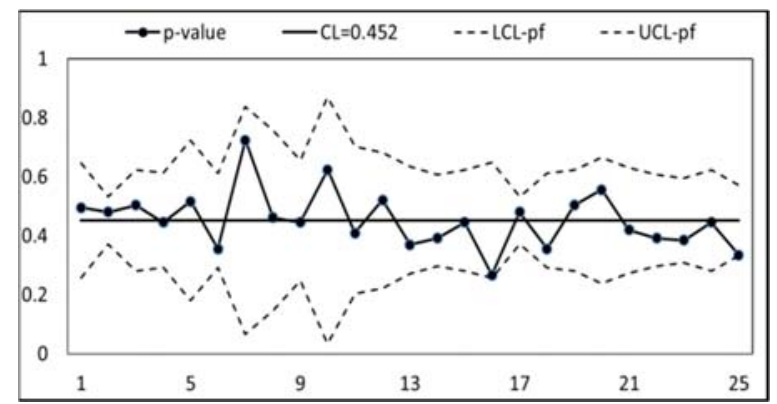

Fig.7. Fuzzy p chart

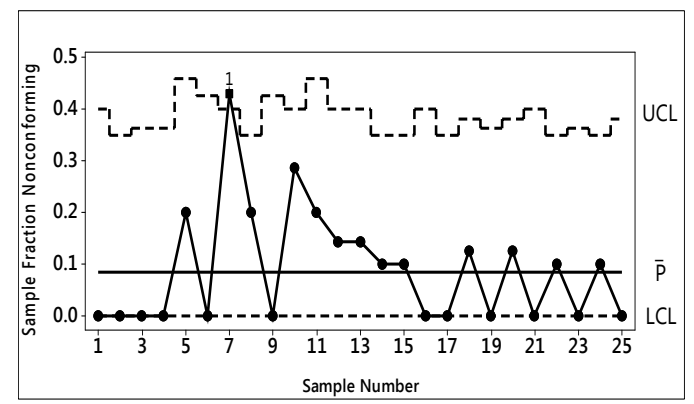

Fig. 8. P control chart for nonconforming keys 
In this section, a $p$ chart for nonconforming keys is presented. This diagram which has been drawn by MINITAB software (Version: 17.1.0) is given to be compared to fuzzy control chart (Fig 8). As can be seen, in order to process analysis with Shewhart control charts, we need to prepare a separate graph for each category.

\section{Table 4}

centerline and control limits for fuzzy $p$ chart

\begin{tabular}{|c|c|c|c|c|c|}
\hline day & p-value & $\sigma$ & $\mathrm{CL}$ & $\mathrm{LCL}_{\mathrm{pf}}$ & $\mathrm{UCL}_{\mathrm{pf}}$ \\
\hline 1 & 0.495 & 0.099 & 0.452 & 0.258 & 0.646 \\
\hline 2 & 0.48 & 0.041 & 0.452 & 0.372 & 0.532 \\
\hline 3 & 0.504 & 0.088 & 0.452 & 0.28 & 0.624 \\
\hline 4 & 0.445 & 0.082 & 0.452 & 0.291 & 0.613 \\
\hline 5 & 0.516 & 0.139 & 0.452 & 0.18 & 0.724 \\
\hline 6 & 0.356 & 0.081 & 0.452 & 0.293 & 0.611 \\
\hline 7 & 0.724 & 0.197 & 0.452 & 0.066 & 0.838 \\
\hline 8 & 0.462 & 0.156 & 0.452 & 0.146 & 0.758 \\
\hline 9 & 0.445 & 0.104 & 0.452 & 0.248 & 0.656 \\
\hline 10 & 0.623 & 0.214 & 0.452 & 0.033 & 0.871 \\
\hline 11 & 0.409 & 0.127 & 0.452 & 0.203 & 0.701 \\
\hline 12 & 0.521 & 0.117 & 0.452 & 0.223 & 0.681 \\
\hline 13 & 0.369 & 0.093 & 0.452 & 0.27 & 0.634 \\
\hline 14 & 0.391 & 0.079 & 0.452 & 0.297 & 0.607 \\
\hline 15 & 0.445 & 0.087 & 0.452 & 0.281 & 0.623 \\
\hline 16 & 0.267 & 0.1 & 0.452 & 0.256 & 0.648 \\
\hline 17 & 0.48 & 0.041 & 0.452 & 0.372 & 0.532 \\
\hline 18 & 0.356 & 0.081 & 0.452 & 0.293 & 0.611 \\
\hline 19 & 0.504 & 0.088 & 0.452 & 0.28 & 0.624 \\
\hline 20 & 0.556 & 0.109 & 0.452 & 0.238 & 0.666 \\
\hline 21 & 0.419 & 0.091 & 0.452 & 0.274 & 0.63 \\
\hline 22 & 0.391 & 0.079 & 0.452 & 0.297 & 0.607 \\
\hline 23 & 0.385 & 0.073 & 0.452 & 0.309 & 0.595 \\
\hline 24 & 0.445 & 0.087 & 0.452 & 0.281 & 0.623 \\
\hline 25 & 0.334 & 0.061 & 0.452 & 0.332 & 0.572 \\
\hline
\end{tabular}

\section{Conclusion and Recommendations}

In the present study, the fuzzy approach has been used for statistical control of an unusual process to reduce the culls. The main purpose of our research is to demonstrate the fuzzy methods applications. The detailed definition of fuzzy membership functions for more compatibility to physical world has been the main challenge for simulation of process control. The correct identification of limitations in inspection process and utilization of proper fuzzy rules for modeling is the second challenge of this research. The results show that the fuzzy technique for dealing with multinomial attribute data works quite well if the problem is defined well. Using one chart containing all categories is more economical than several separate charts. Further to this, the fuzzy charts are more sensitive in giving the alarm for shift in the specified quality levels. It is important when developing a fuzzy model of a process that the care is taken to listen to the experts and get the best model and simulation possible. The aforementioned fuzzy approach has the potential for future use in simulation of unusual process with high-volume production such as metallurgical industry. Wherein, the product is affected not only by the main factors, but by direct effects of temperature and pressure and as a result different quality levels are formed.

\section{References}

Bajpai, N. (2009). Business statistics. Pearson Education India.

Bradshaw, C. W. (1983). A fuzzy set theoretic interpretation of economic control limits. European Journal of Operational Research, 13(4), 403-408.

Cheng, C. B. (2005). Fuzzy process control: construction of control charts with fuzzy numbers. Fuzzy sets and systems, 154(2), 287-303. 
Cox, E. (2005). Fuzzy modeling and genetic algorithms for data mining and exploration. Elsevier.

Du, K. L., \& Swamy, M. N. (2006). Neural networks in a softcomputing framework. Springer Science \& Business Media.

El-Shal, S. M., \& Morris, A. S. (2000). A fuzzy rule-based algorithm to improve the performance of statistical process control in quality systems.Journal of Intelligent \& Fuzzy Systems, 9(3, 4), 207223.

Franceschini, F., \& Romano, D. (1999). Control chart for linguistic variables: a method based on the use of linguistic quantifiers. International Journal of Production Research, 37(16), 3791-3801.

Fryman, M. A. (2002). Quality and process improvement. Cengage Learning.

Gülbay, M., Kahraman, C., \& Ruan, D. (2004). $\alpha$-Cut fuzzy control charts for linguistic data. International Journal of Intelligent Systems, 19(12), 1173-1195.

Gülbay, M., \& Kahraman, C. (2007). An alternative approach to fuzzy control charts: Direct fuzzy approach. Information sciences, 177(6), 1463-1480.

Kahraman, C. (Ed.). (2006). Fuzzy applications in industrial engineering (Vol. 201). Heidelberg: Springer.

Kahraman, C., Tolga, E., \& Ulukan, Z. (1995, October). Using triangular fuzzy numbers in the tests of control charts for unnatural patterns. In Emerging Technologies and Factory Automation, 1995. ETFA'95, Proceedings., 1995 INRIA/IEEE Symposium on (Vol. 3, pp. 291-298). IEEE.

Kanagawa, A., Tamaki, F., \& Ohta, H. (1993). Control charts for process average and variability based on linguistic data. The International Journal of Production Research, 31(4), 913-922.

Kim, S. H. (1992). Statistics and decisions: An introduction to foundations. CRC Press.

Rubinstein, R. Y., \& Kroese, D. P. (2016). Simulation and the Monte Carlo method (Vol. 10). John Wiley \& Sons.

Lighter, D. E., \& Fair, D. C. (2000). Principles and methods of quality management in health care. Jones \& Bartlett Learning.

Misra, K. B. (Ed.). (2008). Handbook of performability engineering. Springer Science \& Business Media.

Montgomery, D. C. (2009). Introduction to statistical quality control. John Wiley \& Sons (New York).

Pandurangan, A., \& Varadharajan, R. (2011). Fuzzy multinomial control chart with variable sample size. International Journal of Engineering Science, 3.

Raz, T., \& Wang, J. H. (1990). Probabilistic and membership approaches in the construction of control charts for linguistic data. Production Planning \& Control, 1(3), 147-157.

Ross, T. J. (2009). Fuzzy logic with engineering applications. John Wiley \& Sons.

Shu, M. H., \& Wu, H. C. (2010). Monitoring imprecise fraction of nonconforming items using p control charts. Journal of Applied Statistics,37(8), 1283-1297.

Starczewski, J. T. (2012). Advanced concepts in fuzzy logic and systems with membership uncertainty (Vol. 284). Springer.

Taleb, H., \& Limam, M. (2002). On fuzzy and probabilistic control charts.International Journal of Production Research, 40(12), 2849-2863.

Timm, N. H. (2002). Applied multivariate analysis. Springer Verlag.

Wang, J. H., \& RAZ, T. (1990). On the construction of control charts using linguistic variables. The International Journal of Production Research, 28(3), 477-487.

Webber, L., \& Wallace, M. (2011). Quality control for dummies. John Wiley \& Sons.

Zimmermann, H. J. (2011). Fuzzy set theory-and its applications. Springer Science \& Business Media.

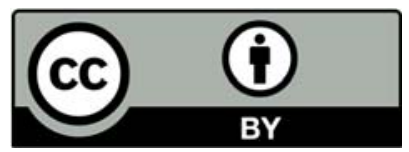

(C) 2017 by the authors; licensee Growing Science, Canada. This is an open access article distributed under the terms and conditions of the Creative Commons Attribution (CC-BY) license (http://creativecommons.org/licenses/by/4.0/). 